\title{
RUMORES Y OTROS MALOS HÁBITOS. EL CONDICIONAL EVIDENCIAL EN ESPAÑOL
}

\author{
FERNANDo BERMúdeZ \\ Universidad de Uppsala \\ fernando.bermudez@moderna.uu.se
}

This paper investigates the so-called "rumor conditional" in Spanish (CR), typical of the journalistic discourse, as in El primer ministro renunciaría en las próximas horas. Its modal features and particularly its evidential properties are discussed from a deictic understanding of evidentiality, based on the work of Bermúdez (2006). CR is compared to another use of the conditional verb form, typical of scientific discourse, with which it is often confused, as in juventud seria el lapso que media entre la madurez fisica y la madurez social. It is proposed that the $\mathrm{CR}$ is a marker of third-hand information, while the scientific conditional indicates second-hand information and cognitive access to the information source. The analysis also functions as indirect evidence of the adequacy of the deictic approach to evidentiality.

Keywords: conditional, evidentiality, deixis, epistemic modality

En este artículo se investiga el llamado "condicional de rumor" (CR) en español, forma típica del discurso periodístico, como en El primer ministro renunciaría en las próximas horas. Los rasgos modales, y particularmente los valores evidenciales de esta forma verbal se discuten a partir de una visión deíctica de la evi- 
dencialidad basada en Bermúdez (2006). El CR se compara con otro uso de la forma verbal condicional, típica del discurso científico, con el que a menudo se confunde, como en juventud sería el lapso que media entre la madurez física y la madurez social. Se propone que el CR es un marcador de información de tercera mano, mientras que el "condicional científico" indica información de segunda mano y acceso cognitivo a la fuente de información. El análisis funciona asimismo como una evidencia indirecta de la adecuación de la visión deíctica de la evidencialidad.

Palabras clave: condicional, evidencialidad, deixis, modalidad epistémica

\section{InTRODUCCIÓN}

Puede afirmarse sin temor a exagerar que el tiempo verbal condicional en español (1-3) es una forma verbal incómoda, de difícil clasificación.

(1) Dijo que llegaría mañana.

(2) Saldría al campo si no estuviera lloviendo.

(3) Tendría frío y por eso estaba temblando.

El condicional de (1) suele interpretarse como un futuro del pasado, mientras que en (2) expresa un significado modal de baja potencialidad; en (3), por su parte, el condicional expresa una inferencia, lo cual involucra un componente modal (probabilidad) y al mismo tiempo evidencial (la información es accedida mediante un proceso cognitivo).

Más allá del mayor o menor interés científico que pueda otorgarse a las consideraciones normativas de la Real Academia Española (RAE), un simple examen de las diferentes ediciones de la Gramática da clara cuenta de la dificultad de su clasificación. En un principio se lo incluyó, 
en la tradición de Nebrija, como una forma del pretérito imperfecto del modo subjuntivo (RAE 1913); más tarde, en un intento de separarla del subjuntivo pero sin confundirla con el indicativo, "modo de la realidad", se creó una categoría modal específica, el modo potencial, que solo incluía el condicional simple y compuesto (RAE 1917; 1931); finalmente, se lo terminó incluyendo dentro del modo indicativo (lo que ya Andrés Bello había hecho en 1847), como un postpretérito o futuro del pasado (RAE 1973; RAE/ASALE 2009). Ninguna otra forma verbal ha experimentado un periplo clasificatorio tan variado como el condicional. Aun en su actual situación relativamente estable dentro del modo indicativo, esta forma verbal continúa ofreciendo dificultades tanto en su descripción como en su clasificación. En efecto, la mera existencia del condicional simple y compuesto puso de manifiesto la necesidad de modificar el modelo reichenbachiano de la descripción del significado de los tiempos verbales añadiendo un segundo punto de referencia para justamente poder dar cuenta de su complejidad deíctica (Vikner 1985; Carrasco Gutierrez 2008).

Para complicar aún más el panorama, el condicional posee usos corteses (4) y evidenciales (5-6):

(4) Deberías cortarte el cabello.

(5) Pampita y Benjamín Vicuña esperarían otro hijo. (Clarín, 18/04/2014)

(6) Los jugadores no entrenan y el presidente renunciaría esta noche. (El Diario, 15/01/2014)

En (4), mediante la morfología condicional el hablante atenúa la necesidad expresada por el verbo modal deber, mientras que en (5) y 
(6) el hablante expresa una calificación evidencial, a saber, que la fuente de la información expresada en el enunciado no es el propio hablante. Por último, usos como el de (5) y (6) suelen ser considerados como galicismos de aparición relativamente reciente y restringidos al discurso periodístico, y por lo tanto se los suele calificar como normativamente incorrectos y/o marginales.

El presente trabajo examina los usos evidenciales del condicional, como en (5-6), tratando de ofrecer una descripción de su significado y, de manera tangencial, discutir los argumentos a los que se ha acudido para cuestionar la corrección de su uso. Los ejemplos presentados provienen de diferentes variantes del español, lo que sugiere que las conclusiones aquí extraídas son válidas para el español en general, más allá de las diferencias que diferentes variantes presentan tanto en el uso de las formas verbales como en la expresión de significados evidenciales.

\section{El CONDICIONAL EN ESPAÑOL}

Una cantidad considerable de estudios han abordado el tema del condicional, ya sea específicamente (Lozano 1988; García Fajardo 2000; Azzopardi 2011; 2013; Hennemann 2014) o como una parte dentro del estudio general de los tiempos verbales del español (Bull 1960; Seco 1972; Rojo 1990; Porto Dapena 1991; Veiga 1991; Pérez 1998; Rojo \& Veiga 1999; Carrasco Gutiérrez 2008; Hennemann 2013 entre otros). En la mayoría de los casos se señala el valor de futuro en el pasado (1) y el hipotético (2) como nucleares, nombrando más o menos de paso los otros usos "modales" como los de (4-6). En dos de los trabajos más 
recientes dedicados en profundidad al condicional, Azzopardi (2011; 2013), apoyándose en la evolución diacrónica de la forma -ría a partir de la perífrasis latina original, le asigna un valor nuclear de ulterioridad en el pasado: la desinencia -ía de imperfecto de indicativo expresaría la referencia a una situación enunciativa anterior al momento de la enunciación mientras que el morfema $-r$ - estaría situando el acontecimiento como ulterior. ${ }^{1}$ A partir de esta definición básica intenta hacer una descripción exhaustiva de los usos del condicional, e identifica dos tipos diferentes: los usos temporales y los usos modales. Entre los usos temporales (posterioridad en el pasado) identifica dos usos específicos, a los que llama subjetivo o de ulterioridad (7) y objetivo o histórico (8):

(7) Pedro dijo que vendría.

(8) Un año más tarde se convertiría en presidente, en 1995.

Entre los usos modales identifica cuatro efectos de sentido, a saber: hipotético (9), atenuativo (10), conjetural (11) y citativo (12):

(9) Si te fueras, estaría contento.

1 Thomas (2012), sin embargo, deriva el significado hipotético, ya presente en el latín, no del valor temporal sino del valor de necesidad presente en la perífrasis latina original, compartido con el valor temporal. De esta manera, la propuesta de, entre otros, Azzopardi (2011; 2013) de que el valor de ulterioridad en el pasado es el valor básico de esta forma puede cuestionarse. Otra propuesta, si bien no directamente asociada al condicional, es la de Iatridou (2000), quien relaciona las nociones de pretérito e irrealidad, al afirmar que la morfología asociada al pretérito en realidad expresa una función particular (a saber, la de exclusión) entre un tiempo/mundo tópico y el tiempo/mundo del enunciado. 
(10) ¿Podrías darme un poco de pan?

(11) Cuando lo conocí él tendría veinte años.

(12) Según El País, el presidente estaría en Estados Unidos.

Azzopardi (2013: 340 y ss.) señala, apoyándose entre otros en Thomas (2012) y Company Company (1986) que los valores hipotético (9) y de ulterioridad subjetiva en el pasado (7) existían ya en la perífrasis original latina (derivados del significado inicial de necesidad) y han continuado siendo productivos hasta el presente, mientras que los demás usos serían derivados de estos dos sentidos primitivos. Azzopardi afirma que el primero que aparece es el atenuativo o cortés, con ejemplos tan tempranos como del siglo XIII, seguido del conjetural, que aparece en el siglo XVI. Las primeras apariciones del citativo se remontan al siglo XviII según Azzopardi, y por último los usos históricos no hacen su entrada sino hasta el siglo XIx.

En el siguiente apartado nos detendremos particularmente al uso que Azzopardi llama citativo; primero pasaremos revista a los estudios anteriores sobre el tema y luego presentaremos nuestro análisis desde una visión deíctica de la evidencialidad.

\section{El CONDICIONAL DE "RUMOR"}

El uso del condicional que aquí nos ocupa ha recibido diferentes nombres en la bibliografía en lengua española: condicional de rumor (RAE/ASALE 2009), condicional periodístico (Veiga 1991; Pérez 1998), de información no asegurada (Lapesa 1977; Seco 1998), potencial citativo (Nadal Palazón 2011), condicional citativo (Azzopardi 2013) y condicional epistémico de 
atribución (Kronning 2015). Tales denominaciones rescatan diferentes aspectos tradicionalmente asociados a este uso del condicional, a saber, que se lo encuentra (principalmente) en el discurso periodístico, que marca una fuente de información diferente al hablante y que expresa cierto grado de incertidumbre respecto de lo enunciado. En efecto, un enunciado como (12) remite directamente al discurso periodístico, al tiempo que indica que la información expresada (que el presidente está en Estados Unidos) ha sido recibida de una fuente externa, y al mismo tiempo que el periodista no se hace cargo de su veracidad.

Como se señaló anteriormente, dentro de los trabajos que tratan la forma verbal condicional, el condicional de rumor (en adelante, CR) ha ocupado un lugar periférico. En efecto, cuando se lo nombra suele agrupárselo con otros usos "modales" o "dislocados" (Rojo \& Veiga 1999), dándole un status de curiosidad lingüística. Casos típicos son los de Gili Gaya (1962), que da cuenta de este uso en una nota al pie, Rojo \& Veiga (1999), que lo menciona como un par de líneas de anotación en cuerpo de letra menor, o el de la Nueva gramática de la lengua española (RAE/ASALE 2009), que le dedica escasas siete líneas, y presentándolo como "una variante del condicional de conjetura" (es decir, una subvariedad de casos como 11).

Asimismo, este uso del condicional ha tenido un bajo prestigio desde el punto de vista normativo: típicamente se lo suele acusar de "galicismo" 2

${ }^{2}$ Los libros de estilo de muchos periódicos en lengua española españoles cuestionan y hasta prohíben el uso del CR:

La posibilidad en el pasado no es, sin embargo, un hecho dudoso, no garantizado, ni un rumor. Este uso del condicional de indicativo es francés. Se incurre, pues, en galicismo cuando se escriben frases como éstas: 'el ministro de Agricultura podría estar dispuesto' 
(Lázaro Carreter 2001; Seco 1998: 350), se lo presenta como delimitado al discurso periodístico (Veiga 1991; Lázaro Carreter 2001) y de aparición reciente. Estos tres aspectos se encuentran claramente relacionados: la crítica normativa afirma que el CR no es original del castellano sino que es copiado del francés por periodistas hispanos, lo cual implica que su uso no es más antiguo que la época de difusión de la prensa escrita; de hecho, Azzopardi (2013: 343) fecha la primera aparición del CR en el siglo XVIII, cuando los primeros periódicos aparecen en España. En los últimos años, sin embargo, se han relativizado algunos de estos aspectos. Kronning (2002: 563) afirma que lejos de ser una propiedad del discurso periodístico, el CR se encuentra por ejemplo en el discurso científico e histórico. Asimismo presenta ejemplos de este uso tan antiguos como del siglo xvi, lo cual al mismo tiempo estaría cuestionando indirectamente el hecho de que fuera un galicismo, dado que si este uso existe en la lengua castellana desde fecha tan antigua, y en diferentes tipos de discurso, podría afirmarse que es un rasgo que pertenece al castellano por derecho propio. ${ }^{3}$ Ahora bien, ¿cuáles son realmente las propiedades temporales, evidenciales y modales del condicional de rumor? ¿Se encuentra

...; 'el obispo habría establecido...'; ·según diversas fuentes, habrían sido detenidos siete grapos ...'; [...] El uso del condicional en ese tipo de frases queda terminantemente prohibido en el periódico. Además de incorrecto gramaticalmente, resta credibilidad a la información. (El País 2003)

El condicional con el sentido de suposición es un barbarismo sintáctico, originado en una inexacta traducción del francés, que atenta contra la credibilidad de la información, sobre todo cuando la encabeza en el título, bajada o resumen. Sólo por esto este solecismo es inadmisible. (La Nación 1997)

3 Martines (2015) encuentra asimismo este uso en el catalán ya en el siglo XIII. 
efectivamente restringido al discurso periodístico o, como afirma Kronning (2002), se encuentra en muchos otros tipos de discurso? En los siguientes apartados trataremos de responder estas preguntas.

\subsection{El CR en el discurso periodístico}

En el presente apartado echaremos una mirada al "condicional de rumor" y trataremos de establecer su semántica, y en particular sus propiedades evidenciales.

En cuanto a la deixis temporal, se suele afirmar que el significado nuclear del condicional es un futuro del pasado (Azzopardi 2011; 2013, entre otros). Sin embargo, el CR, como puede notarse en (56), no se relaciona explícitamente con pasado alguno, y temporal y aspectualmente se comporta más bien como el tiempo verbal presente y no como un futuro; esto es, los estados se interpretan como coetáneos con el momento de la enunciación (13) mientras que los procesos se interpretan con referencia futura (14) o como hábitos (15). ${ }^{4}$

(13) Simon soltó su bomba: Humala tendría un hijo extramatrimonial. ( $E l$ Comercio, 27/3/2014)

(14) Los jugadores no entrenan y el presidente renunciaría esta noche. (El Diario, 15/01/2014)

4 Rojo \& Veiga (1999: 2916) incluyen el cr como un "uso dislocado" de la forma verbal condicional, que surgiría al eliminar el vector de posterioridad asociado a la forma verbal condicional o al reemplazarlo por un vector de simultaneidad. Para una visión más detallada esta interpretación, basada en la teoría temporalista de Rojo, véase Rojo (1974). 
(15) El presidente trabajaría con los movimientos sociales. (El Potosí, $31 / 03 / 2015)$

¿Qué decir de su significado evidencial? La denominación de condicional "de rumor" sugiere que se trata de información que no es de primera mano y además de origen desconocido, o al menos no explícito. Contra esta descripción del significado evidencial del condicional es fácil reaccionar, dado que la fuente de información puede muy bien estar explícita en el contexto inmediato, como puede verse en los siguientes ejemplos:

(16) Igor Shkuro, de treinta y dos años, autor ayer del secuestro de un avión italiano con 101 pasajeros y 11 tripulantes a bordo que volaba entre Argel y Roma y que terminó sin mayores incidentes, tendría sus facultades mentales perturbadas, según se informó hoy en Roma. (ABC, 27/09/1982) (17) Nols estaría considerando, según el propio Le Pen, la creación en Bélgica de un Frente Nacional de extrema derecha, similar al francés. (El País, 01/10/1984)

En efecto, en (16) se alude a una fuente, aunque oculta en la forma pasiva, y en (17) se presenta una fuente específica: Le Pen. La cuestión es entonces si se puede afirmar que se está expresando un rumor aun cuando se hace referencia a la fuente. Claramente es una cuestión de definición, esto es, a qué se llama "rumor". ¿Es solamente rumor cuando la fuente es inespecífica como en "parece que/dicen que/dizque Humala tiene un hijo extramatrimonial", o se considera también rumor cuando se reconoce una fuente directa más o menos específica, como en "todos 
los que lo conocen dicen que Humala tiene un hijo extramatrimonial" o "Mi peluquero dice que Humala tiene un hijo extramatrimonial"?

Sea como fuere, se puede apreciar una diferencia notable en las propiedades evidenciales del CR en relación con otras formas verbales que también introducen información de segunda mano, como por ejemplo el pluscuamperfecto: ${ }^{5}$

(18) Dice que por allá no hay ninguna... ninguna librería. No hay ninguna. Vamos a ver. No sé si lo... si la inaugurarán pronto, porque el otro día habían ido a marcar la mercadería. Dice que tenían mucha mercadería ya comprada, pero no sé cómo les irá. (Habla Culta: Buenos Aires: M27 B)

La elección del pluscuamperfecto en (18) (el hablante bien podría haber elegido el perfecto simple: fueron) se explica porque del contexto se entiende que el hablante no ha sido testigo directo de la información que se expresa: concretamente, el hablante, al utilizar el pluscuamperfecto, expresa explícitamente que no estaba allí cuando fueron a marcar la mercadería, sino que sus dichos se basan en información de segunda mano. Ahora bien, el enunciado de (18) nunca podría tomarse por un rumor, aun cuando la fuente no es completamente explícita (se puede sin embargo deducir que la fuente concuerda con el sujeto de dice), en este sentido la interpretación es completamente diferente de (16) o (17), aun cuando en (17) la fuente es identificada con una persona

5 Para una caracterización del pluscuamperfecto como marcador evidencial, véase Bermúdez $(2008 ; 2011)$. 
determinada (Le Pen). Pero ¿cómo definir esta diferencia intuitiva, que hace que los enunciados con condicional puedan interpretarse como rumor mientras que los enunciados con pluscuamperfecto, que también marca que la fuente de la información no es el propio hablante, no reciban tal interpretación? Para responder esta pregunta daremos un rodeo por el dominio semántico de la evidencialidad.

Definimos evidencialidad aquí como el dominio semántico relacionado con la fuente de la información expresada en un enunciado. Pero ¿cómo se estructura internamente la categoría semántica de la evidencialidad? Una variante del siguiente esquema, que sigue en parte a Willett (1988: 57), suele ser el más utilizado entre los estudios de la evidencialidad. Según esta posición, los subdominios de la evidencialidad podrían esquematizarse como sigue, en función del tipo de evidencia que el hablante declara poseer: ${ }^{6}$

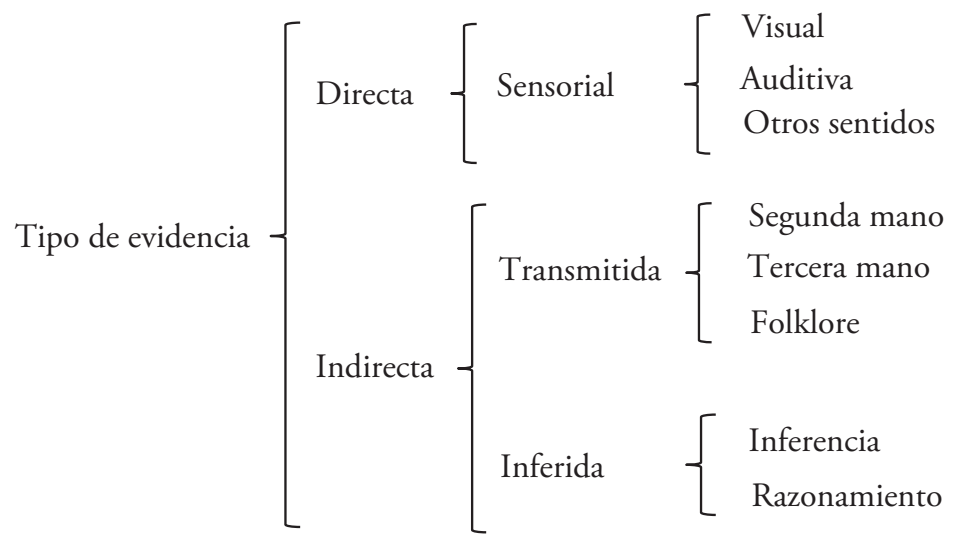

Figura 1. Los subdominios de la evidencialidad

6 Para otras clasificaciones de la evidencialidad, véanse, entre otros, Frawley (1992), Rooryck (2001), Plungian (2001; 2010), Aikhenvald (2004; 2014) y Brugman (2015). 
Para aclarar el esquema de la Figura 1 podemos decir que la evidencia directa sensorial se refiere a aquellos casos en los que el hablante afirma haber presenciado la situación directamente por medio de la vista, el oído u otros sentidos. ${ }^{7}$ La evidencia indirecta inferida se refiere a casos en los que el hablante no tiene acceso directo a la situación descrita pero sí posee contacto directo con huellas o rastros de esa situación que le permiten inferir lo que ocurre o ha ocurrido (inferencia) o conoce algo que le permite deducir que es probable que la situación se dé o se haya dado (razonamiento); en otras palabras, la diferencia entre inferencia y razonamiento en la Figura 1 se basa en el tipo de premisas que conducen a la conclusión. Por último, la evidencia indirecta transmitida, que es la que nos interesa particularmente aquí, se refiere a los casos en los que el hablante no tiene acceso ni a la situación ni a indicios de la situación, sino que la información le ha sido transmitida por otra persona. Un caso especial dentro de la evidencia indirecta transmitida es aquella información que no proviene de los dichos de una persona específica, sino que proviene de lo que en términos generales puede llamarse folklore o saber popular.

7 Dentro de la evidencia directa suele incluirse a veces la evidencia directa endofórica (Tournadre 1996; Plungian 2001), que se refiere a casos en los que el hablante describe entidades inaccesibles a los sentidos, como los deseos, los sueños, las intenciones y los estados mentales en general, es decir, a aquellas situaciones en las que la evidencia sensorial es imposible, pero en las que el hablante de todos modos aduce evidencia directa, ya que no se trata de inferencias ni de información transmitida por otra persona. Este dominio endofórico no existía en la clasificación original de Willett (1988), sin embargo, Tournadre (1996) ha argumentado convincentemente que en las lenguas del Tíbet existen marcadores específicos que indican si la fuente de información es endofórica. 
A partir del esquema de la Figura 1 podemos establecer entonces la diferencia entre el significado evidencial del pluscuamperfecto y el del condicional, concretamente mediante las subcategorías de la información transmitida. En efecto, en (18), repetido aquí como (19), el hablante declara no haber sido testigo de la situación, sino que ha recibido la información de una fuente, pero se sobreentiende que tal fuente posee la información de primera mano; esto es, ha sido testigo de la situación. Por el contrario, en (17), repetido aquí como (20), la fuente aducida (Le Pen) necesariamente ha recibido a su vez la información de una fuente (tal vez el propio Nols, pero la cadena bien puede ser mucho más larga):

(19) Dice que por allá no hay ninguna... ninguna librería. No hay ninguna. Vamos a ver. No sé si lo... si la inaugurarán pronto, porque el otro día habían ido a marcar la mercadería. Dice que tenían mucha mercadería ya comprada, pero no sé cómo les irá.

(20) Nols estaría considerando, según el propio Le Pen, la creación en Bélgica de un Frente Nacional de extrema derecha, similar al francés. ( $E l$ Pais, 01/10/1984)

Es decir, ambos señalan que la fuente de información es externa, pero mientras el pluscuamperfecto presenta una información como de segunda mano, el condicional establece una distancia mayor entre el hablante y la fuente de información, ya que esta se presenta como de (al menos) tercera mano.

En cuanto al significado modal del CR, existen varias afirmaciones no totalmente coincidentes. Azzopardi (2013: 343) afirma que el CR "indica 
que el contenido tiene cierto grado de incertidumbre", Vatrican (2010; 2014: 262) postula que el CR presenta un estado de cosas como "hipotético, parafraseable por puede que" y muchos libros de estilo condenan su uso porque "expresa [...] una afirmación de cuya veracidad se duda" (La voz de Galicia 2009: 64), y “resta credibilidad a la información” (El País 2003: 105). La adecuación de estas afirmaciones no es, sin embargo, evidente. En el ejemplo (21) es fácil pensar que el periodista duda de la veracidad de la información (de hecho, al final del artículo el mismo periodista afirma que "Expertos conocedores de las centurias y cuartetos de Nostradamus, aseguraron que el pasaje es un invento de los usuarios del internet"), pero por el contrario, en (22) el periodista parece estar completamente seguro de la información, lo que se transluce a partir del contexto, en el uso del verbo revelar y el hecho de que afirma que la información fue confirmada por otras fuentes relevantes.

(21) Gangnam Style estaría vinculado con el fin del mundo. El famoso astrólogo Nostradamus habría predicho que el famoso Gangnam Style del rapero coreano Psy, sería un presagio del fin del mundo. (Últimas noticias, 05/12/2012)

(22) Uno de los siete jueces de la Corte Suprema de Justicia de la Nación, Enrique Petracchi, pediría licencia en el tribunal por una grave enfermedad, según reveló ayer el portal Infobae y confirmaron fuentes judiciales. (Clarín, 17/03/2014)

En otras palabras, las suposiciones sobre la veracidad de la información transmitida serían más bien el resultado de una evaluación del lector a partir 
de datos del contexto, pero no incluidas en la semántica de la forma verbal, en concordancia con Dendale (1993: 174), que, refiriéndose al francés, afirma que la toma de partido por el grado de veracidad de la información es una consecuencia pragmática (aunque al mismo tiempo afirma que tal rasgo de incertidumbre se asigna por defecto). Coincidimos entonces con Kronning $(2002 ; 2004)$ en que la aportación modal del CR se trata de lo que él llama modalización cero, es decir, que el hablante, si bien renuncia a hacerse cargo de la veracidad de la información expresada, al mismo tiempo no la pone en duda. En el caso concreto de (20), el hablante no se hace cargo de la veracidad de la información (que Nols está considerando la creación de un Frente Nacional en Bélgica), pero tampoco la pone en duda. Por su parte, en (21) el lector posiblemente interpreta la información como altamente dudosa y en (22) como verdadera, pero solamente por la incidencia del contexto, y no como resultado de la mera aparición de la forma verbal condicional: el periodista simplemente renuncia a hacerse cargo de la información, más allá de que la considere más o menos segura.

En resumen, el llamado condicional de rumor en el discurso periodístico tiene las mismas propiedades temporales que el presente del indicativo y, al utilizarlo, el hablante, en el dominio modal, renuncia a hacerse cargo de la veracidad de la información expresada en el enunciado y, evidencialmente, califica al enunciado como de, al menos, tercera mano.

\subsection{El discurso científico}

Como se ha señalado anteriormente, Kronning (2002) rechaza la idea de que el CR, que él denomina condicional epistémico de atribución, se 
restrinja al discurso periodístico, y da pruebas claras de que en el discurso científico e histórico existe un uso frecuente de este condicional cuya función es declarar una fuente de información externa. En (23) podemos ver un ejemplo de tal uso:

(23) Robert Nisbet examina breve y rigurosamente los temas centrales del conservadurismo - historia, tradición, propiedad, autoridad, libertad y religión- y dirige sus críticas tanto al colectivismo como al individualismo radical. Finalmente, aborda la crisis del conservadurismo, que estaría causada en buena medida por su adopción en la escena política de principios del liberalismo económico y del Estado del bienestar.

En efecto, en (23) el condicional indica que la fuente de la información expresada en el enunciado (que la crisis del conservadurismo está causada por la adopción de principios del liberalismo económico y del Estado de bienestar) es externa; en concreto, expresa que la fuente es Robert Nisbet. Al mismo tiempo, al utilizar el condicional el hablante renuncia a hacerse cargo de la veracidad de la información: compárese (23) con el enunciado alternativo (24), donde el hablante no asigna la información a una fuente externa y al mismo tiempo se hace cargo de su veracidad; o, en otras palabras, donde afirma que la crisis del conservadurismo tiene mayormente tales causas:

(24) Robert Nisbet examina breve y rigurosamente los temas centrales del conservadurismo - historia, tradición, propiedad, autoridad, libertad y religión-y dirige sus críticas tanto al colectivismo como al individualis- 
mo radical. Finalmente, aborda la crisis del conservadurismo, que está causada en buena medida por su adopción en la escena política de principios del liberalismo económico y del Estado del bienestar.

Tenemos entonces en (23) un uso del condicional que indica información transmitida, que presenta las mismas propiedades temporales que el tiempo presente y que en el plano modal expresa la renuncia a hacerse cargo de la veracidad de la información expresada en el enunciado. Esto parecería estar confirmando la tesis de Kronning (2002) de que el CR no solo existe en el discurso periodístico, y que la denominación condicional de rumor es, pues, poco adecuada, dado que evidentemente el enunciado de (23) no se trata de un rumor.

Pero ¡es realmente el condicional de (23) equivalente en términos evidenciales al condicional de (17)? Para aclarar esta cuestión analizaremos estos ejemplos a la luz de una visión algo diferente de la evidencialidad.

En trabajos anteriores (Bermúdez 2006; 2008; 2011), hemos argumentado a favor de una visión deíctica de la evidencialidad, basada en el modelo de la deixis espacial. La deixis espacial trata de la perspectivización del espacio físico a partir de un centro que define el punto de vista; o, en otras palabras, la ubicación relativa de objetos en el espacio en relación con un centro deíctico (típicamente el hablante). Tal ubicación puede bien definirse mediante tres parámetros continuos, a saber: dirección, distancia y puntos de referencia. Uno de estos puntos de referencia, el centro deíctico (típicamente identificado con el hablante) es de vital importancia, dado que constituye el origen de la perspectivización. De esta manera, una expresión como detrás de en el lápiz está detrás de los libros es deíctica dado 
que organiza el espacio a partir del punto de vista del hablante, y puede representarse de la siguiente manera: 'el lápiz está en la misma dirección que el punto de referencia los libros desde la perspectiva del hablante, pero a una distancia mayor'. Gráficamente (Figura 2):

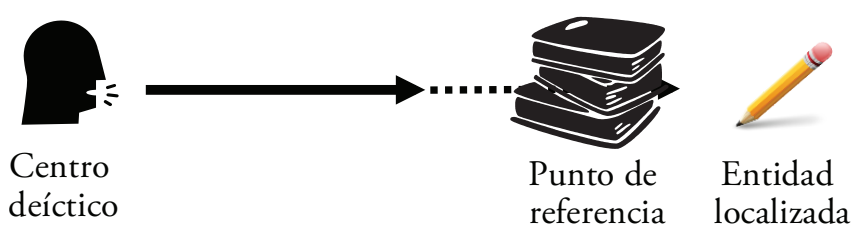

Figura 2. Representación deíctica del significado de detrás de

Del mismo modo que la deixis espacial trata de la perspectivización del espacio en relación con un centro deíctico (típicamente el hablante), según nuestra posición la evidencialidad trata asimismo de la ubicación de la información expresada en los enunciados en relación con un centro deíctico típicamente identificado con el hablante. De tal manera, nuestra hipótesis es que el dominio semántico de la evidencialidad puede definirse a partir de los mismos tres parámetros que definen la deixis espacial: dirección, distancia y puntos de referencia. Ahora bien, dado que en el caso de la evidencialidad como categoría deíctica no se trata de ubicar objetos en el espacio sino información con respecto a individuos, estos parámetros se interpretan metafóricamente. El parámetro de la dirección se reinterpreta entonces como dirección de la información: hacia el hablante (el caso de la evidencia sensorial, donde el hablante recibe una impresión) o desde el hablante (el caso de la evidencia inferida, inferencia o razonamiento, donde 
el hablante elabora cognitivamente la información). ${ }^{8}$ Importante es destacar aquí que estos parámetros son continuos: en un extremo se situaría la evidencia sensorial visual, que en principio se trataría de pura impresión sensorial sin ninguna elaboración cognitiva, y en el otro se ubicaría el razonamiento del modelo de Willett de la Figura 1, que se trataría de pura elaboración cognitiva sin rastros de impresiones sensoriales. Entre estos extremos se pueden, sin embargo, identificar puntos intermedios, como la inferencia, que si bien es básicamente elaboración cognitiva se basa al menos parcialmente en estímulos sensoriales, o la evidencia endofórica (como los deseos, los sueños, etc.) que están a medio camino entre impresión sensorial y elaboración cognitiva. Llamamos a este parámetro el modo de acceso a la información. El segundo parámetro de la deixis espacial, la distancia, se trata en el caso del dominio evidencial de distancia a la fuente de información. También este parámetro es continuo y la gradación va entre un extremo que sería la máxima cercanía posible a la fuente de información (información de primera mano, donde la fuente es el propio hablante) y otro extremo que sería la máxima lejanía a la fuente de información (como por ejemplo el rumor: dicen que dicen que dicen...). Puntos intermedios aquí serían información de segunda mano o de tercera mano. Por último, los puntos de referencia del modelo deíctico ya no son objetos en el espacio como en la deixis espacial sino individuos con o sin acceso a la información. Este parámetro se extiende desde un extremo que sería el caso donde el hablante aduce tener acceso exclusivo a la fuente de información (y, por lo tanto, presenta la información como nueva para el interlocutor) hasta el

8 Para un análisis de la evidencialidad también basado en criterios deícticos pero en muchos aspectos diferente al nuestro véase Frawley (1992). 
extremo donde el hablante postula que la información es de acceso universal (como en el caso del folklore, donde se postula que todos los integrantes de una comunidad lingüística, por el mero hecho de pertenecer a ella, tienen acceso a tal información). También aquí hay espacios intermedios, y particularmente importante y productivo es el caso en el que el hablante aduce acceso compartido a la información; esto es, cuando expresa que tanto hablante como oyente tienen acceso a la información expresada en el enunciado (es decir, cuando se postula información compartida). ${ }^{9}$ Llamamos a este parámetro acceso a la fuente de información. Gráficamente:

Modo de acceso

a la información:

Distancia a la fuente de información:

Acceso a la fuente de información:

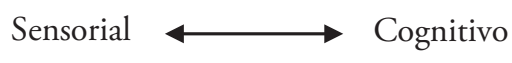

Interna

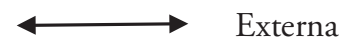

Exclusivo

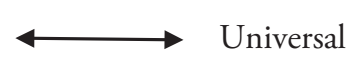

Figura 3. El dominio semántico de la evidencialidad

9 El valor mirativo (esto es, las marcas de sorpresa que el hablante expresa cuando el conocimiento contradice sus propias expectativas) tendría su explicación dentro de este parámetro, dado que la sorpresa que el valor mirativo involucra podría definirse en términos de acceso a la información: en el mirativo el hablante señala su propia falta de acceso a la información. El mirativo como valor evidencial es, sin embargo, discutido: Chafe (1986), por ejemplo, lo incluye como un valor evidencial, mientras que Plungian (2001) sugiere que el mirativo es en todo caso un marcador modal pero no evidencial, dado que no marca el modo de acceso a la información sino en qué medida el hablante está preparado para percibir lo que percibe; DeLancey (1997; 2001) por su parte, propone que el mirativo debe ser reconocido como una categoría gramatical y semántica independiente, separada tanto de la modalidad epistémica como de la evidencialidad. 
Estos tres parámetros delinean, pues, un espacio en el que los diferentes valores evidenciales pueden ubicarse (para una revisión de las ventajas de este sistema frente a otras propuestas de clasificación, véase Bermúdez 2006). Un aspecto importante de este modelo es que estos parámetros son independientes, lo cual predice todo un espectro de combinaciones posibles y casos limítrofes.

A partir de este modelo podemos volver a considerar ahora la diferencia entre el CR y el pluscuamperfecto que señalamos en el apartado anterior: tanto el pluscuamperfecto de (18) como el condicional de (17) establecen una distancia hacia la fuente de información, pero el pluscuamperfecto presenta la información como "no de primera mano", mientras que el CR la presenta como "ni de primera ni de segunda mano". Es decir, el pluscuamperfecto se ubicaría en un punto intermedio del continuo fuente interna $\leftrightarrow$ externa, mientras que el condicional coloca la fuente de información a una distancia mayor respecto del centro deíctico representado por el hablante. Gráficamente (Figura 4):

Distancia a la Interna fuente

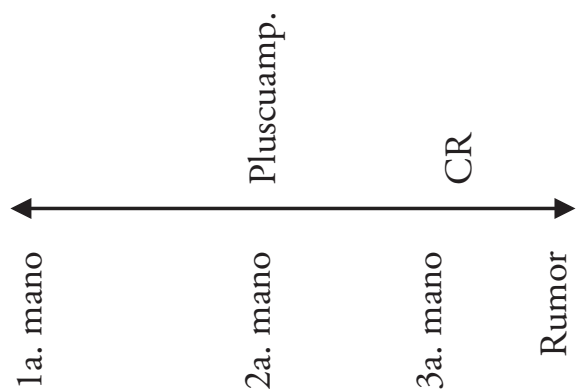

Figura 4. El cR y el pluscuamperfecto y la distancia a la fuente de información 
Esto es análogo a lo señalado con respecto al parámetro del modo de acceso a la información: si bien tanto el razonamiento como la inferencia y la evidencia endofórica se alejan del polo del modo de acceso sensorial, lo hacen en distintos grados, donde el razonamiento es el más alejado, la inferencia, aunque es principalmente elaboración cognitiva, se sitúa más cerca del polo sensorial, mientras que la evidencia endofórica se sitúa a medio camino entre el modo de acceso sensorial y el cognitivo.

Ahora bien, ¿`ómo clasificar el caso de (23), repetido aquí como (25)?

(25) Robert Nisbet examina breve y rigurosamente los temas centrales del conservadurismo -historia, tradición, propiedad, autoridad, libertad y religión- y dirige sus críticas tanto al colectivismo como al individualismo radical. Finalmente, aborda la crisis del conservadurismo, que estaría causada en buena medida por su adopción en la escena política de principios del liberalismo económico y del Estado del bienestar.

Claramente el hablante atribuye la información a una fuente externa, y esa fuente es Robert Nisbet. O sea, si bien la información se atribuye a una fuente externa, tal fuente es relativamente cercana al hablante; es decir, es información de segunda mano. Por el contrario, el CR en el discurso periodístico presenta la información como (al menos) de tercera mano: 'alguien dice que alguien dice (que alguien dice que alguien dice...) X'. Aquí tenemos entonces la primera diferencia entre el "condicional periodístico" de (12-17) y el "condicional científico" de (25): mientras el primero presenta la información como de tercera mano, el otro la presenta como de segunda mano, y atribuyéndola a una 
instancia específica, lo cual no es necesario en el caso del condicional periodístico, como puede verse en (15).

Sin embargo, las diferencias no acaban allí. Como señalamos anteriormente, el modelo de la Figura 3 implica que los tres parámetros son independientes y que por lo tanto dos significados evidenciales pueden diferir a lo largo de uno o más parámetros. Nuestra hipótesis es que este es el caso aquí. Si analizamos en detalle el enunciado de (25) veremos que el hablante no solo está diciendo que esta información proviene de Nisbet, sino también que la información expresada (que la crisis del conservadurismo en gran medida está ocasionada por la adopción de los principios del liberalismo y el Estado de bienestar) no es meramente una cita, sino más bien es una interpretación, una elaboración cognitiva de las palabras originales de Nisbet. Y esto es típico de los casos del condicional evidencial en el discurso científico. Veamos otros casos análogos:

(26) Este concepto [moratoria social] adhiere implícitamente a ciertos límites vinculados con la condición de juventud: esta etapa transcurriría entre el final de los cambios corporales que acaecen en la adolescencia y la plena integración a la vida social que ocurre cuando la persona forma un hogar, se casa, trabaja, tiene hijos. O sea juventud sería el lapso que media entre la madurez física y la madurez social. [...] Desde esta perspectiva, sólo podrían ser jóvenes los pertenecientes a sectores sociales relativamente acomodados. Los otros carecerían de juventud. (Margulis \& Urresti 1998: 5)

(27) Como ya señalamos anteriormente, según la posición temporalista los ejemplos (22) y (23) se explican como "usos especiales" del distanciamien- 
to temporal, que en estos contextos perdería su impronta temporal y se reinterpretaría como distanciamiento cortés. (Reyes 1990, entre otros)

En (26), el autor analiza críticamente los conceptos de moratoria social y juventud (que no le pertenecen sino que los refiere a otros autores) e interpreta ciertas características de estos conceptos (por ejemplo que la juventud, según estos autores, sería la etapa entre la madurez física y la madurez social) y ciertas consecuencias (por ejemplo, que los sectores de nivel socioeconómico más bajo carecerían de juventud). Como en el caso anterior, esta información es asignada a una fuente externa, pero al mismo tiempo se marca que es producto de una (mayor o menor) elaboración cognitiva de parte del escritor del artículo. En otras palabras: el enunciador interpreta el discurso atribuido a una fuente externa. Tal elaboración cognitiva no existe en los casos del condicional evidencial en el discurso periodístico. Lo mismo ocurre en (27): el autor no solo asigna esas afirmaciones a una fuente externa, sino que en realidad propone que esas afirmaciones se deducen de los dichos de esa fuente.

En resumen, los datos aquí presentados parecen mostrar que el condicional típico del discurso periodístico es un marcador evidencial que señala información de tercera mano, mientras que el condicional "científico" es un marcador evidencial que señala información de segunda mano y acceso cognitivo. ${ }^{10}$

10 Esto puede explicar la variedad terminológica señalada anteriormente: aquellos que solo se concentran en el condicional periodístico lo llaman preferentemente de rumor, dado que su especificidad es justamente marcar la información como de tercera mano, mientras que Kronning, que intenta unificar los usos periodísticos y los usos científicos, lo llama de atribución, 
Tal diferencia tiene consecuencias en la interpretación modal de esta forma lingüística: el condicional periodístico, como ya hemos señalado, indica información de tercera mano, lo que la hace mucho más sensible a aspectos contextuales, como, por ejemplo, si existe una fuente explícita o no, la credibilidad de tal fuente, etc. Sin embargo, más allá de tal interpretación del lector, que puede acercarse más (22) o menos (21) a la certeza, el enunciador renuncia de todos modos a hacerse cargo de veracidad de la información. Por el contrario, el condicional científico atribuye la información a una fuente directa y específica (por lo que no presenta esa característica de "rumor"), pero al mismo tiempo es producto de una elaboración cognitiva, lo que hace que si bien la renuncia a hacerse cargo de la veracidad de la información subsiste, al mismo tiempo se suma un nivel de complejidad: el enunciador sí se hace cargo de la elaboración cognitiva que ha aplicado al discurso original de la fuente aludida.

\section{4. ¿UNO O DOS CONDICIONALES DE ATRIBUCIÓN?}

La pregunta que surge en este punto de la discusión es si estos dos usos del condicional son meras variantes de un mismo fenómeno o si se trata de dos tipos diferentes. La respuesta de aquellos que han estudiado el condicional evidencial en francés sería, seguramente, que se trata del mismo fenómeno. Sin embargo, hay ciertas razones para creer que, al menos en español, se trata de dos fenómenos en parte diferentes.

puesto que lo que ambos tienen en común es el hecho de que la información es atribuida a una fuente externa, sea esta específica o no, sea de segunda o tercera mano. 
En primer lugar, el aspecto más obvio: el hecho de que tengan diferentes propiedades evidenciales: mientras que el "condicional científico" expresa información de segunda mano y modo de acceso cognitivo, el periodístico expresa información de tercera mano. Por otro lado, si se tratara del mismo fenómeno, sería improbable que la reacción normativa solo se hubiera producido contra los casos aparecidos en el discurso periodístico y nunca contra los casos aparecidos en el discurso científico, aún más al tener en cuenta que estos son mucho más frecuentes que los condicionales periodísticos y tienen una historia mucho más larga. Por último, el condicional científico se encuentra conceptualmente cerca del circunstancial hipotético, y puede pensarse que se deriva de este, como puede verse en los siguientes ejemplos, adaptados de (26):

(28) Según Margulis, sólo podrían ser jóvenes los pertenecientes a sectores sociales relativamente acomodados. Los otros carecerían de juventud.

(29) Desde esta perspectiva, sólo podrían ser jóvenes los pertenecientes a sectores sociales relativamente acomodados. Los otros carecerían de juventud.

(30) Si se definiera juventud como moratoria social, sólo podrían ser jóvenes los pertenecientes a sectores sociales relativamente acomodados. Los otros carecerían de juventud.

El condicional periodístico, por el contrario, a pesar de ser más reciente, no guarda rastros claros de su origen en el castellano, sino que aparece, según Azzopardi (2011) en el siglo XVIII, con el comienzo de la prensa española. Esto abre el interrogante, que debería estudiarse más 
en profundidad, de si el condicional periodístico, como postulan los normativistas, no es verdaderamente un calco del francés, introducido por la prensa espańola a partir del prestigio de la prosa francesa del Siglo de las Luces, mientras el "condicional científico" es un desarrollo autóctono a partir del condicional hipotético, lo cual explicaría las características que lo diferencian del condicional periodístico. En efecto, el hecho de que el condicional científico exprese información de segunda mano (y no de tercera mano) puede explicarse a partir de la relación original del condicional con la prótasis del período hipotético, lo mismo que su tendencia a tener una fuente explícita más o menos determinada. De su origen en el período hipotético puede también fácilmente derivarse la tendencia a expresar modo de acceso cognitivo y su aparición típica en textos científicos.

\section{Resumen y CONCLUSIONES}

Hemos estudiado las características semánticas del "condicional de rumor", estableciendo que su valor evidencial es expresar información de tercera mano. De acuerdo con Kronning proponemos que el valor modal de esta forma es la renuncia a hacerse cargo de la veracidad de la información, pero sin ponerla en duda. El eventual grado de incertidumbre que se presenta en enunciados concretos es una inferencia contextual, pero no un significado inscrito en la forma verbal condicional. Luego hemos comparado el condicional de rumor con el condicional evidencial típico del discurso científico y hemos encontrado ciertas diferencias esenciales, como por ejemplo que el condicional científico expresa información de 
segunda mano y modo de acceso cognitivo a la fuente de información, lo que acarrea consecuencias en su significado modal epistémico, a saber, que si bien por un lado expresa la renuncia a hacerse cargo de la veracidad de la información presentada en el enunciado, el hablante al mismo tiempo sí se hace cargo de la elaboración cognitiva realizada sobre el discurso atribuido. Estas diferencias abren el interrogante de si estos dos usos del condicional no tienen un origen diferente: mientras el condicional del discurso científico, con sus propiedades específicas, puede derivarse del condicional hipotético, el condicional de rumor del discurso periodístico bien puede haber sido un calco del uso francés, introducido en el siglo XVIII. Sin embargo, para corroborar esta hipótesis sería necesario realizar un estudio diacrónico que permita establecer el origen de ambos usos.

\section{REFERENCIAS}

Aikhenvald, Alexandra. 2004. Evidentiality. Oxford: Oxford University Press.

Aikhenvald, Alexandra. 2014. The grammar of knowledge: A crosslinguistic view of evidentials and the expression of information source. En Aikhenvald, Alexandra \& Dixon, Robert M. W. (eds.), The grammar of knowledge: A cross-linguistic typology, 1-51. Oxford: Oxford University Press.

Azzopardi, Sofie. 2011. Le Futur et le Conditionnel: valeur en langue et e ets de sens en discours. Analyse contrastive espagnol / français. Montpellier: Université Paul Valéry-Montpellier III. (Tesis doctoral.) 
Azzopardi, Sofie. 2013. Valor aspectuo-temporal y usos modales del condicional a la luz de la noción de gramaticalización. En Cabedo Nebot, Adrián \& Aguilar Ruiz, Manuel \& López-Navarro Vidal, Elena (eds.), Estudios de lingüistica: investigaciones, propuestas y aplicaciones, 337-347. Valencia: Tecnolingüística.

Bello, Andrés. 1970 [1847]. Gramática de la lengua castellana. 8a ed. Buenos Aires: Editorial Sopena Argentina.

Bermúdez, Fernando. 2006. Evidencialidad. La codificación lingüistica del punto de vista. Estocolmo: Universidad de Estocolmo. http:// su.diva-portal.org/smash/get/diva2:199511/FULLTEXT01. (Consultado el 30-05-2016.)

Bermúdez, Fernando. 2008. Había sido o no había sido, he ahí la cuestión. Pluscuamperfecto y evidencialidad en castellano. Studia Neophilologica 80(2). 203-222.

Bermúdez, Fernando. 2011. El pluscuamperfecto como marcador evidencial en castellano. En Hernández Socas, Elia \& Sinner, Carsten \& Wotjak, Gerd (eds.), Estudios de tiempo y espacio en la gramática española, 43-62. Frankfurt am Main: Peter Lang.

Brugman, Claudia. 2015. Characterizing evidentiality. Linguistic Typology 19(2). 201-237.

Bull, William. 1960. Time, tense, and the verb: A study in theoretical and applied linguistics, with particular attention to Spanish. Berkeley: University of California Press.

Carrasco Gutiérrez, Ángeles. 2008. Los tiempos compuestos del español. Formación interpretación y sintaxis. En Carrasco Gutiérrez, Ángeles 
(ed.), Tiempos compuestos y formas verbales complejas, 15-64. Madrid: Iberoamericana Editorial.

Chafe, Wallace. 1986. Evidentiality in English Conversations and Academic Writing. En Chafe, Wallace \& Nichols, Johanna (eds.), Evidentiality: The linguistic coding of epistemology, 261-272. Norwood: Ablex.

Company Company, Concepción. 1986. Los futuros en el español medieval. Sus orígenes y su evolución. Nueva Revista de Filología Hispánica 34(1). 48-108.

DeLancey, Scott. 1997. Mirativity: the grammatical marking of unexpected information. Linguistic Typology 1. 33-52.

DeLancey, Scott. 2001. The mirative and evidentiality. Journal of Pragmatics 33. 339-464.

Dendale, Patrick. 1993. Le conditionnel de "l'information incertaine": marqueur modal ou marqueur évidentiel? En Hilty, Gerold (ed.), Actes du XXe Congrès International de Linguistique et Philologie Romanes, vol. 1, 165-176. Tubinga: Francke Verlag.

El País. 2003. Libro de estilo. 18 a ed. Madrid: Ediciones El País. Frawley, William. 1992. Linguistic Semantics. Hillsdale: Lawrence Erlbaum.

García Fajardo, Josefina. 2000. Los valores del... ¿"pospretérito”? Nueva Revista de Filología Hispánica 48(1). 25-49.

Gili Gaya, Samuel. 1962 [1943]. Curso superior de sintaxis española. Barcelona: VOX.

Hennemann, Anja. 2013. A context-sensitive and functional approach to evidentiality in Spanish or why evidentiality needs a superordinate category. Frankfurt am Main: Peter Lang. (Tesis doctoral.) 
Hennemann, Anja. 2014. The evidential use of the Spanish imperfect and the conditional in journalistic contexts. Studia Neophilologica 86(2). 183-200.

Iatridou, Sabine. 2000. The grammatical ingredients of counterfactuality. Linguistic Inquiry 31(2). 231-270.

Kronning, Hans. 2002. Le conditionnel 'journalistique': médiation et modalisation épistémiques. Romansk forum 16(2). 561-575.

Kronning, Hans. 2004. Modalite et mediation epistemique. En Delamotte-Legrand, Régine (ed.), Les médiations langagières. Volume 1, Des faits de langue aux discours: Actes du Colloque International La Médiation, Marquages en Langue et en Discours, vol. 1, 35-65. MontSaint-Aignan: Presses universitaires de Rouen.

Kronning, Hans. 2015. El condicional epistémico 'de atribución' en francés, italiano y español: aspectos diafásicos, diatópicos y diacrónicos. En Jeppesen Kragh, Kirsten \& Lindschouw, Jan (eds.), Les variations diasystématiques et leurs interdépendances dans les langues romanes, 507-518. Estrasburgo: Éditions de linguistique et de philologie.

La Nación. 1997. Manual de estilo y ética periodística. Buenos Aires: Espasa Calpe.

Lapesa, Rafael. 1977. Tendencias y problemas actuales de la lingüística espańola. En Lapesa, Rafael (ed.), Comunicación y lenguaje, 203-229. Madrid: Karpos.

La voz de Galicia. 2009. Libro de estilo. http://www.prensaescuela.es/ attachments/article/51/LEVoz\%203.6.pdf. (Consultado el 30-052016.)

Lázaro Carreter, Fernando. 1990. El idioma del periodismo, ¿lengua especial? En García, Pedro \& Gómez, Alberto (eds.), El idioma español 
en las agencias de prensa, 25-44. Madrid: Fundación Germán Sánchez Ruipérez.

Lázaro Carreter, Fernando. 2001. El dardo en la palabra. Barcelona: Galaxia Gutenberg.

Lozano, Anthony G. 1988. The semantics of the Spanish conditional in Discourse. Hispania 71. 675-680.

Margulis, Mario \& Urristi, Marcelo. 1998. La construcción social de la condición de juventud. En Cubides, Humberto \& Laverde Toscano, María Cristina \& Margulis, Mario \& Valderrama, Carlos Eduardo (eds.), Viviendo a toda: Jóvenes, territorios culturales y nuevas sensibilidades, 3-21. Santa Fe de Bogotá: Fundación Universidad Central / Departamento de Investigaciones.

Martines, Josep. 2015. Semantic change and intersubjectification: The origin of reprise evidential conditional in Old Catalan. Catalan Journal of Linguistics 14. 79-111.

Nadal Palazón, Juan Gabriel. 2011. El discurso ajeno en los titulares periodisticos. Salamanca: Universidad de Salamanca. (Tesis doctoral.) Pérez, María Rosa. 1998. Realizaciones del contenido modal irrealidad en el sistema verbal español. En Luquet, Gilles (ed.), Travaux de linguistique hispanique: actes du VIIe Colloque de linguistique hispanique organisé à la Sorbonne les 8, 9 et 10 février 1996, 385-399. París: Presses de la Sorbonne Nouvelle.

Plungian, Vladimir. 2001. The place of evidentiality within the universal grammatical space. Journal of Pragmatics 33. 349-357.

Plungian, Vladimir 2010. Types of verbal evidentiality marking: An overview. En Diewald, Gabriele \& Smirnova, Elena (eds.), Linguistic realization of evidentiality in European languages, 15-58. Berlín: De Gruyter. 
Porto Dapena, José Álvaro. 1991. Del indicativo al subjuntivo. Valores y usos de los modos del verbo. Madrid: Arco Libros.

Real Academia Española. 1917. Gramática de la lengua española. Madrid: Espasa Calpe.

Real Academia Española. 1931. Gramática de la lengua española. Madrid: Espasa Calpe.

Real Academia Española. 1973. Esbozo de una nueva gramática de la lengua española. Madrid: Espasa Calpe.

Real Academia Española \& Asociación de Academias de la Lengua Espańola. 2009. Nueva gramática de la lengua española. Madrid: Espasa Libros.

Reyes, Graciela. 1990. Valores estilísticos del imperfecto. Revista de Filología Española 70. 45-70.

Rojo, Guillermo. 1974. La temporalidad verbal en español. Verba 1. 68-149.

Rojo, Guillermo. 1990. Relaciones entre temporalidad y aspecto en el verbo español. En Bosque, Ignacio (ed.), Tiempo y aspecto en español, 17-43. Madrid: Cátedra.

Rojo, Guillermo \& Veiga, Alexandre. 1999. El tiempo verbal: los tiempos simples. En Bosque, Ignacio \& Demonte, Violeta (eds.), Gramática descriptiva de la lengua española, vol. 2, 2867-2929. Madrid: Espasa Calpe.

Rooryck, Johan. 2001. Evidentiality, part I. Glot International 5. 125-133. Seco Manuel. 1972. Gramática esencial del español. Introducción al estudio de la lengua. Madrid: Espasa Calpe. 
Seco, Manuel. 1998. Diccionario de dudas y dificultades de la lengua española. Madrid: Espasa Calpe.

Thomas, Jean-François. 2012. Ultériorité dans le passé et éventualité en latin. Faits de langues 40(2). 13-20.

Tournadre, Nicolas. 1996. Comparaison des systèmes médiatifs de quatre dialectes tibétains tibétain central, ladakhi, dzonkha et amdo. En Guentchéva, Zlakta (ed.), L'énonciation médiatisée, 195-213. París: Peeters.

Vatrican, Axelle. 2010. La modalité et le conditionnel de rumeur en français et en espagnol. Modèles linguistiques 31(62). 83-94.

Vatrican, Axelle. 2014. Usos y valores modales del condicional en español. Archivum 64. 239-274.

Veiga, Alexandre. 1991. Condicionales, concesivas y modo verbal en español. Santiago de Compostela: Universidad de Santiago de Compostela. Vikner, Sten. 1985. Reichenbach revisited: one, two or three temporal relations? Acta Linguistica Hafniensia 19(2). 81-98.

Willett, Thomas. 1988. A cross-linguistic survey of the grammaticization of evidentiality. Studies in Language 12. 51-97.

Fecha de recepción de manuscrito original: 27 de enero de 2016 Fecha de recepción de manuscrito revisado: 13 de julio de 2016 Fecha de aceptación: 13 de julio de 2016 